\title{
Acromegaly presenting as subarachnoid haemorrhage in a 76 year old woman
}

\author{
K. Beard ${ }^{1}$, I.C. Macdougall ${ }^{1}$ and W.M.H. Behan ${ }^{2}$ \\ ${ }^{1}$ University Department of Medicine and ${ }^{2}$ Department of Pathology, Western Infirmary, Glasgow, UK.
}

\begin{abstract}
Summary: Acromegaly usually presents in middle age with the gradual onset of classical signs and symptoms. We report the case of an elderly woman who presented with subarachnoid haemorrhage due to infarction of a pituitary adenoma and rupture into the subarachnoid space.
\end{abstract}

\section{Introduction}

Gradual changes in facial features, together with growth of the head, hands and feet is the usual presenting picture of acromegaly. Less commonly, the presentation may be that of visual field disturbance, usually outer quadrantanopia or, at a later stage, bitemporal hemianopia. Dull frontal headache is often described by acromegalics, although it is seldom the sole presenting feature. Although the pituitary adenoma which produces the syndrome is vascular, haemorrhage or infarction in this lesion is uncommon and rupture into the subarachnoid space is rare (Wakai et al. 1981).

We present the case of an elderly woman whose acromegaly was undiagnosed until she presented with subarachnoid haemorrhage due to rupture.

\section{Case report}

A 76 year old retired teacher was admitted to hospital as an emergency with a $7 \mathrm{~d}$ history of lethargy and intermittent throbbing headache. This started at the vertex, radiated over the occiput and neck, lasted between 1 and $4 \mathrm{~h}$ on each occasion, and was not relieved by simple analgesia. Over the $24 \mathrm{~h}$ before admission the headache had become much more severe and continuous with nausea and vomiting on 4 occasions, and her husband had noted episodes of slurred and confused speech.

The only past medical history of note was that of a corneal ulcer which had resulted in secondary

Correspondence: I.C. Macdougall, B.Sc., M.B., Ch.B., Ward 7C, Gartnavel General Hospital, Great Western Road, Glasgow G12 0YN, UK.

Accepted: 2 January 1985 glaucoma and for which she had recently undergone a right sided tarsorrhaphy. She had also been hypertensive for the last few years, and was being treated with a thiazide diuretic.

On examination, several features of acromegaly were noted including prognathism, macroglossia and large hands and feet. She was pyrexial $\left(37.9^{\circ} \mathrm{C}\right)$, the pulse was $130 / \mathrm{min}$ and the blood pressure $250 /$ $105 \mathrm{mmHg}$. Nuchal rigidity and a positive Kernig's sign were noted, but apart from slight drowsiness and generalized hyperreflexia, there were no abnormal neurological signs. The left optic disc was clear, but the tarsorrhaphy prevented inspection of the right. Similarly it was difficult to assess the visual fields, but there appeared to be no gross defect in that of the left eye by confrontation.

A lumbar puncture was performed and yielded blood stained fluid under normal pressure. The cerebrospinal fluid (CSF) glucose level was $1.1 \mathrm{mmol} / 1$ (blood glucose $7.6 \mathrm{mmol} / \mathrm{l}$ ), and microscopy showed numerous white cells, mostly polymorphs, with a grossly elevated white cell (WBC): red cell (RBC) ratio of $1: 15$ (normal 1:1000). Xanthochromia was noted but no organisms could be detected. Infection was suspected in view of the cellularity and glucose level, and she was treated with penicillin and chloramphenicol. Her condition deteriorated rapidly and she died on the day after admission, a repeat lumbar puncture having confirmed the previous findings.

The skull X-ray on admission was of poor quality and no useful information was gained regarding the pituitary fossa. Analysis of an ante-mortem blood sample yielded the following results: human growth hormone (HGH) $51 \mathrm{mU} / 1$ (variable normal range, but this level is consistent with active acromegaly - MRC reference no. 66/217), cortisol $385 \mathrm{nmol} / \mathrm{l}$ (normal $330-770 \mathrm{nmol} / \mathrm{l}$ ), $\mathrm{T}_{4} 69 \mathrm{nmol} / \mathrm{l}$ (normal $54-142 \mathrm{nmol} / \mathrm{l}$ ), 
TSH $3.6 \mathrm{mU} / 1$ (normal $<6 \mathrm{mU} / \mathrm{l}$ ), $\mathrm{LH} 1.3 \mathrm{IU} / \mathrm{l}$ (normal 23-70 IU/1), FSH <1 IU/l, (normal 30-100 IU/l).

\section{Post-mortem findings}

A post-mortem examination revealed discoloration at the base of the brain with subarachnoid haemorrhage. A large purplish-red nodule with attached blood clot was seen to protrude $1.5 \mathrm{~cm}$ above the sellar diaphragm. The sella was expanded to 8 times normal size and on sagittal section, an encapsulated tumour was visible, extending below the floor to replace most of the sphenoid bone (Figure 1). On microscopy, this tumour was found to be an eosinophil adenoma comprising sheets of uniform cells with occasional bizarre giant forms but no mitoses. A large central haemorrhagic infarct was present with distended veins containing organized thrombus and extending to the subarachnoid space by a narrow slit in the tumour. Around this area, dense focal aggregates of neutrophil leucocytes were found and the entire surface was covered with organizing subarachnoid haemorrhage with neutrophilic infiltration. The adenoma was encapsulated laterally but below it had breached the dura to invade the sphenoid air spaces and above it was demarcated from a thin rim of normal pituitary tissue. The tumour was strongly positive for growth hormone when reacted with antibody to this hormone and examined by the PAP immunoperoxidase technique.

The brain itself showed dark red meningeal blood in relation to the hypothalamus, cerebellum and brain stem, considered to be of several days duration. A concave depression in the hypothalamic region was associated with distortion and flattening of the floor of the third ventricle, the optic nerves, chiasm and optic tracts. On coronal section, an organizing haematoma was found in the third ventricle at the level of the mamillary bodies. The thyroid and both adrenals were small but histology was unremarkable, and the ovaries had been removed many years previously. Apart from macroglossia, there was no other organomegaly.

\section{Discussion}

This is a distinctly unusual case because of both its clinical presentation and its pathological findings. Acromegaly does not often present at such an advanced age, most eosinophil adenomas giving rise to acromegaly between 30 and 50 years of age. This patient suffered no symptoms of acromegaly and neither she nor her family had noticed any change in appearance suggestive of the diagnosis, any such changes having presumably been attributed to 'just old age'.

Pituitary apoplexy is a recognized complication of

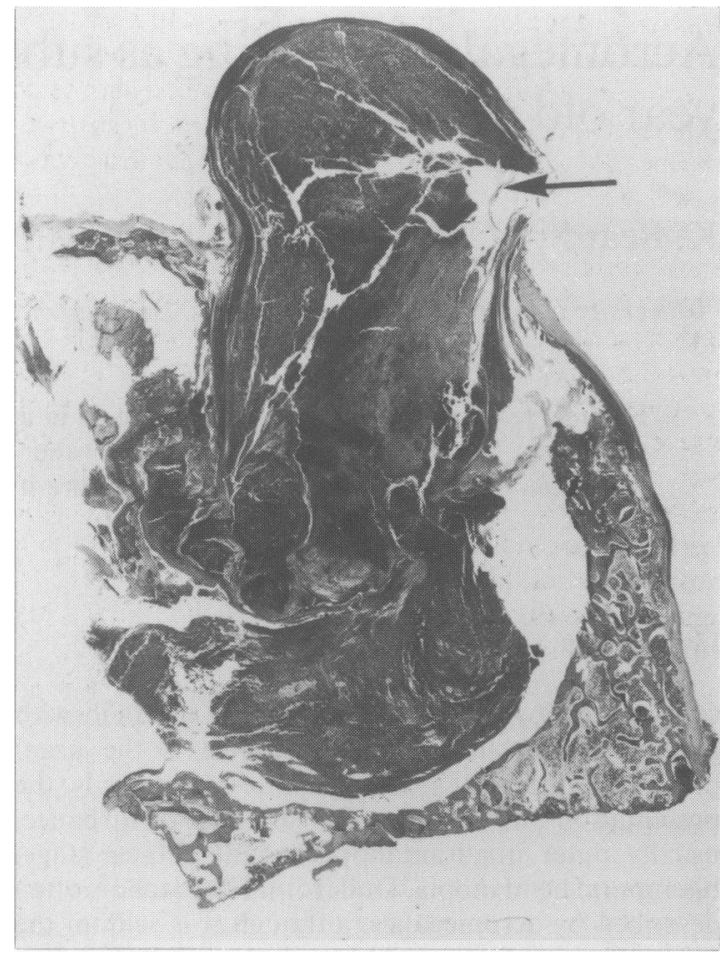

Figure 1 Pituitary fossa, $\mathbf{H} \& \mathrm{E} \times 3$. Tumour has expanded the fossa and infiltrated deeply into sphenoid bone. Anteriorly, the arrow indicates site of rupture from the central area of haemorrhagic necrosis into the subarachnoid space.

acromegaly, and although the mechanism is not completely clear, it has been suggested that the tumour mass suddenly enlarges due to spontaneous haemorrhage or haemorrhagic necrosis within the tumour tissue. These adenomas are very vascular but subarachnoid haemorrhage is a rare presenting picture.

Wakai et al. (1981) reviewed a large series of 560 cases of pituitary adenoma operated on over a period of 30 years. Haemorrhage into the tumour did occur in $16.6 \%$ of cases but extension into the subarachnoid space was rare, being found in only 3 cases $(0.5 \%)$. Jefferson (1940) in his presidential address to the Section of Neurology of the Royal Society of Medicine reported a large study of 'invasive' adenomas, stating that he knew of only 3 cases, including one of his own, where subarachnoid haemorrhage had occurred. Kirschbaum \& Chapman (1948) reported a case similar to ours in that acromegaly had not been diagnosed before the patient presented with a fatal subarachnoid haemorrhage. The main differences were that their patient was a young man aged 35 , and in retrospect it 
was clear that the features of acromegaly had been present for many years. A different presentation was described by Mohanty et al. (1979) where 2 episodes of subarachnoid haemorrhage were followed by surgical removal of a chromophobe adenoma with subsequent good recovery.

There was some diagnostic confusion and the CSF results deserve comment. The high WBC: $\mathrm{RBC}$ ratio, together with the low glucose level, initially suggested a diagnosis of meningitis particularly since neither is found in acute subarachnoid haemorrhage. However, xanthochromia and the absence of organisms were more in favour of a diagnosis of chronic or recurrent subarachnoid haemorrhage. The appearance of numerous leucocytes in the CSF following chronic subarachnoid haemorrhage is due to the production of various chemotactic factors, and the fall in CSF glucose is a consequence of the great metabolic activity of these cells.

Acute ACTH deficiency is an important cause of death in pituitary apoplexy, and steroid replacement is

\section{References}

JEFFERSON, G. (1940). Extrasellar extension of pituitary adenomas. Proceedings of the Royal Society of Medicine, 33, 433.

KIRSCHBAUM, J.D. \& CHAPMAN, B.M. (1948). Subarachnoid haemorrhage secondary to a tumour of the hypophysis with acromegaly. Annals of Internal Medicine, 29, 536. often necessary. Although this patient showed no clinical or biochemical features of adrenal insufficiency, the morning cortisol level of $385 \mathrm{nmol} / 1$, while within the normal range, could be regarded as inadequate in the context of an acute stressful illness. It is, therefore, probably appropriate to give all these patients steroid cover until it can be shown that their endogenous cortisol production is adequate.

Post mortem examination revealed significant compression of the optic pathways, and visual signs or symptoms might have been expected, although these may have been masked by the previous tarsorrhaphy or overshadowed at presentation by the severity of the terminal illness. Occasionally, a large pituitary adenoma may not produce visual upset because, in $10 \%$ of individuals, the optic chiasm lies behind or in front of an enlarging tumour (Jefferson, 1940). Furthermore, the optic chiasm lies $2 \mathrm{~cm}$ above the sellar diaphragm so that a certain amount of upward expansion is possible before compression of the optic pathway occurs.

MOHANTY, S., THACKER, A.K., KATIYAR, B.C., MISRA, S., RAO, C.J. \& RAO, S.N. (1979). Recurrent subarachnoid haemorrhage in pituitary adenoma, Journal of the Association of Physicians of India, 27, 539.

WAKAI, S., FUKUSHIMA, T., TERAMOTO, A. \& SANO, K. (1981). Pituitary apoplexy: its incidence and clinical significance, Journal of Neurosurgery, 55, 187. 